\title{
Dopamine Modulation of Perisomatic and Peridendritic Inhibition in Prefrontal Cortex
}

\author{
Wen-Jun Gao, Yun Wang, and Patricia S. Goldman-Rakic \\ Department of Neurobiology, Yale University School of Medicine, New Haven, Connecticut 06510
}

\begin{abstract}
The computations underlying cognitive functions are performed by a diversity of interactions between interneurons and pyramidal neurons that are subject to modulatory influences. Here we have used paired whole-cell recording to study the influence of dopamine on local inhibitory circuits involving fast-spiking (FS) and non-FS cells, respectively. We found that dopamine depressed inhibitory transmission between FS interneurons and pyramidal neurons but enhanced inhibition between non-FS interneurons and pyramidal cells. FS inhibitory transmission exhibited properties associated with presynaptic action at $\mathrm{D}_{1}$ receptors that were not evident in non-FS inhibitory connections. In addition, FS and non-FS interneurons differed morphologically, forming contacts on the perisomatic and peridendritic domains, respectively, of their pyramidal cell targets. These findings provide evidence for both a dual mode of inhibition in prefrontal circuitry and circuit-dependent modulation by dopamine.
\end{abstract}

Key words: dual whole-cell recording; GABA; local circuits; interneurons; dopamine; cortical slice

\section{Introduction}

The dopamine (DA) innervation of the prefrontal cortex has been implicated both in the modulation of normal cognitive processes, most particularly working memory, and in numerous neurobiological diseases including Parkinson's disease, agerelated memory decline, and schizophrenia (Gotham et al., 1988; Davis et al., 1991; Volkow et al., 1998; Mattay et al., 2002). The prefrontal cortex receives a rich dopamine innervation from the ventral tegmental area, forming synapses onto both pyramidal cells and interneurons (Sesack et al., 1995; Krimer et al., 1997). We have shown previously that recurrent excitatory transmission between pyramidal cells in the prefrontal cortex is presynaptically depressed by dopamine acting through the $\mathrm{D}_{1}$ receptor (Gao et al., 2001). However, pyramidal cell excitability is also powerfully modulated by inhibitory interneurons (Buhl et al., 1994; Thomson and Deuchars, 1997; Somogyi et al., 1998; Xiang et al., 1998). Indeed, inhibitory processes have been shown recently to have an essential role in sculpting the spatial tuning and temporal dynamics of prefrontal neurons, because they are engaged in mediating the working memory functions of the brain (Wilson et al., 1994; Rao et al., 1999, 2000; Constantinidis et al., 2002).

Inhibitory neurons in the cortex have been differentiated on the basis of their firing patterns in response to depolarizing current pulses and their patterns of dendritic and axonal arborizations (Kawaguchi, 1995; Kawaguchi and Kubota, 1997; Somogyi et al., 1998; Gupta et al., 2000). Although there is as yet no universally agreed on classification of cortical interneurons, it is widely accepted that fast-spiking (FS) interneurons can be distinguished from non-FS cells such as regular spiking (RS), low-

Received Aug. 30, 2002; revised Nov. 25, 2002; accepted Nov. 26, 2002.

This work was supported by National Institute of Mental Health Grant MH44866/MH38546. We thank Anita Begovic for her expert technical support. We are grateful to Drs. T. Koos, W. R. Chen, M. F. Yeckel, S. D. Antic, L. Negyessy, and X. J. Wang for reading this manuscript and for their critical comments.

Correspondence should be addressed to Dr. P. S. Goldman-Rakic, Department of Neurobiology, Yale University School of Medicine, 333 Cedar Street, New Haven, CT 06510. E-mail: patricia.goldman-rakic@yale.edu. Copyright $\odot 2003$ Society for Neuroscience $\quad 0270-6474 / 03 / 231622-09 \$ 15.00 / 0$ threshold spiking (LTS), and late-spiking (LS) cells (Kawaguchi and Kubota, 1997; Gibson et al., 1999). In addition, it has been established that FS inhibitory neurons preferentially innervate the soma or the axonal initial segment of pyramidal cell targets to control action potential initiation, whereas other interneurons primarily regulate dendritic excitability and the efficacy of excitatory inputs (Freund and Buzsaki, 1996; Somogyi et al., 1998).

The effects of dopamine on inhibitory neurons have been studied extensively in the striatum and nucleus accumbens (for review, see Nicola et al., 2000; Bracci et al., 2001) and in subicular neurons (Behr et al., 2000) with extracellular stimulation methods. These studies have revealed that DA can exert significant effects on the activity of individual cells in the striatum and nucleus accumbens by a number of different mechanisms, including modulation of voltage-dependent conductances and effects on excitatory and inhibitory synaptic transmission. Using similar methods, the actions of dopamine on inhibitory transmission in the cerebral cortex indicate that dopamine also inhibits evoked IPSCs in the prefrontal cortex (Gonzalez-Islas and Hablitz, 2001; Seamans et al., 2001). However, considering the physiological and morphological diversity of interneurons in the neocortex (Kawaguchi, 1995; Kawaguchi and Kubota, 1997; Gupta et al., 2000), it is possible that the effects of dopamine are not uniform on all interneuron subtypes. To examine this possibility, we have used paired whole-cell recording of synaptic connections followed by morphological analysis to identify the presynaptic origin of unitary IPSPs. Here we report evidence that dopamine modulates inhibitory transmission in a circuit-dependent manner.

\section{Materials and Methods}

Slice preparation and physiological recording. Methods for slice preparation and whole-cell recording from visualized neurons have been described previously (Gao et al., 2001). In brief, 300- $\mu \mathrm{m}$-thick horizontal slices from young adult ferret (3-4 months of age) prefrontal cortex were cut with a microslicer in ice-cold oxygenated artificial CSF (ACSF) containing (in mM): $124 \mathrm{NaCl}, 2.5 \mathrm{KCl}, 1.25 \mathrm{NaH}_{2} \mathrm{PO}_{4}, 2 \mathrm{CaCl}_{2}, 1 \mathrm{MgSO}_{4}, 26$ 
$\mathrm{NaHCO}_{3}$, and 10 dextrose, $\mathrm{pH}$ 7.4. The slices were incubated in ACSF at $35^{\circ} \mathrm{C}$ for $1 \mathrm{hr}$ and then kept at room temperature until being transferred to the recording chamber. Slices submerged in the recording chamber were perfused with oxygenated and prewarmed ACSF $(2 \mathrm{ml} / \mathrm{min})$. The recordings were conducted at $32-34^{\circ} \mathrm{C}$. Dual whole-cell recordings in current-clamp mode were used for analysis of connections between interneurons and pyramidal cells (NP-P). The resistances of patch pipettes were 5-10 $\mathrm{M} \Omega$ and filled with intracellular solution containing (in $\mathrm{mM}$ ): $114 \mathrm{~K}$-gluconate, $6 \mathrm{KCl}, 0.5 \mathrm{CaCl}_{2}, 0.2$ EGTA, 4 ATP-Mg, $10 \mathrm{HEPES}, \mathrm{pH}$ 7.25, and $0.3 \%$ biocytin (Molecular Probes, Eugene, OR). The signals were amplified and filtered at $2 \mathrm{kHz}$ in bridge-balance mode and acquired on a computer at sampling intervals of $20-100 \mu \mathrm{sec}$ through a DigiData 1200B interface using software pClamp 8.1 (Axon Instruments, Foster City, CA). Access resistance was monitored continuously during recording.

Data analysis. The average IPSP amplitude and SDs of 20-40 traces were measured between the $10 \mathrm{msec}$ interval before the onset of the IPSP and a $5 \mathrm{msec}$ interval at the IPSP peak using Clampfit software (Axon Instruments). The IPSP amplitude was thus defined as the difference between two window averages. Background noise was also measured in the same manner but with the two average windows shifted to $\sim 30 \mathrm{msec}$ before the elicited response (Stricker et al., 1996).

Three analyses were used to assess possible presynaptic and/or postsynaptic mechanisms. First, the percentage of synaptic failure to the evoked presynaptic spike was determined individually for each recording. Failure was defined as an event in which the IPSP amplitude was below the limit of $1.6 \times$ noise (rms). Second, we obtained a paired-pulse ratio (PPR) by measuring the ratio of the first two successive responses (second IPSP to first IPSP) of five responses to pulses given at an interval of $100 \mathrm{msec}(10 \mathrm{~Hz})$. Third, a coefficient of variation (CV) of IPSP amplitude was calculated as described by Kullman (1994). The mean and SD (Mean ${ }_{\text {IPSP }}$ and $\mathrm{SD}_{\text {IPSP }}$ ) were calculated for the IPSP amplitudes recorded during 40 successive sweeps in most cases (27 of 30; 90\%). The CVs for control and during dopamine application were therefore computed as $\mathrm{SD}_{\mathrm{IPSP}} / \mathrm{Mean}_{\mathrm{IPSP}}$. Other measurements included IPSP latency, 20-80\% rise time, decay time constant $(\iota)$, resting membrane potential, and spike threshold of interneurons. The time constant was fit from the repolarization curve of unitary IPSPs by using a standard exponential formula in Clampfit (Axon Instruments). Membrane potentials were not corrected for liquid junction potentials. The data are analyzed by either ANOVA or Student's $t$ test and are presented as mean \pm SE.

To examine whether dopamine modulation is cell-type specific, all of our presynaptic interneurons were classified into FS and non-FS groups. FS interneurons were easily identified by their narrow action potentials, deep and brief afterhyperpolarization (AHP), and high firing rates (100$150 \mathrm{~Hz}$ ) with little or no frequency adaptation. The non-FS class included RS, LTS, and LS, per the criteria described by Kawaguchi (1995) and Gibson et al. (1999). LTS neurons had broader spikes, pronounced adaptation of firing frequency, and more specifically, low-threshold spikes when depolarized from more negative potentials. At threshold stimuli level, RS cells fired regular spikes with prominent firing spike adaptation and wide spikes (usually half-width of $>0.6 \mathrm{msec}$ ) (Kawaguchi, 1995; Gibson et al., 1999).

Drug application. Because dopamine can depolarize or hyperpolarize both pyramidal neurons and interneurons, especially depolarizing FS interneurons (Zhou and Hablitz, 1999), we recorded the IPSPs at the subthreshold potential level $(-47$ to $-62 \mathrm{mV}$; average, $-52.9 \mathrm{mV})$. However, the membrane potential was always kept constant before and during dopamine application for individual experiments. Dopamine was applied either in puff through a glass pipette (tip diameter, $\sim 1-2 \mu \mathrm{m}$; concentration, $0.1-10 \mathrm{~mm}$; pressure, $\left.6.9-13.8 K_{\mathrm{p}}\right)$ or by bath $(10-30 \mu \mathrm{M})$ with addition of the antioxidant ascorbic acid $(10 \mu \mathrm{M}) . \mathrm{A} \mathrm{D}_{1}$-specific dopamine receptor agonist [2,3,4,5-tetrahydro-7,8-dihydroxy-1-phenyl1H-3-benzazepine (SKF 38393), 20-40 $\mu \mathrm{M}$ with $10 \mu \mathrm{M}$ ascorbic acid] and antagonist $[R(+)$-7-chloro-8-hydroxy-3-methyl-1-phenyl-2,3,4,5tetrahydro-1H-3-benzazepine hydrochloride (SCH 23390), 10-20 $\mu \mathrm{M}$ ] and a $\mathrm{D}_{2}$-specific agonist (quinpirole, $15-30 \mu \mathrm{M}$ ) and antagonist (raclopride, $10-20 \mu \mathrm{M}$ ) were bath perfused. To examine the specificity of dopamine receptor modulation, other antagonists, such as the $\alpha-2$ adrenergic receptor antagonists Yohimbine (100 nM) or Prozasin (100 nM) (Marek and Aghajanian, 1999) and the $\mathrm{GABA}_{\mathrm{B}}$ receptor antagonist 3-N[1-(S)-(3,4-dichlorophenyl)ethyl]amino-2-( $S$ )-hydroxypropyl-Pbenzyl-phosphinic acid (CGP55845; $1 \mu \mathrm{M}$ ) were also bath applied. $\mathrm{GABA}_{\mathrm{A}}$ receptors were blocked in some experiments with bath application of bicuculline methiodide ( 5 or $10 \mu \mathrm{M}$ ).

Morphological analysis. Slices were immediately fixed in cold $4 \%$ paraformaldehyde for 3-5 d after recording. The slices were directly reacted in $3 \%$ hydrogen peroxide for $25 \mathrm{~min}$. After thorough rinsing, $\mathrm{ABC}$ reactions were conducted overnight, and then Ni-DAB was reacted on the following day (Tamas et al., 1997; Krimer et al., 2001). The slices were resectioned into either 150 or $60 \mu \mathrm{m}$ sections. The former were directly mounted from phosphate buffer and covered with water-soluble mounting media for reconstruction, whereas the $60 \mu \mathrm{m}$ sections were air-dried and mounted with Permount for cell-type identification and photography. Morphological analysis was conducted by one of the authors (Y.W.), who was blind to their physiological properties. The $150 \mu \mathrm{m}$ sections of recovered cells were viewed and drawn on a MicroBrightField (Williston, VT) camera lucida and classified on the basis of their axonal trajectories, distribution of bouton on somata or dendrites, and dendritic arbors. Correlation of these properties and physiological subtype was performed only after all microscopic study was completed. Selected labeled cells were fully reconstructed with Neurolucida software (MicroBrightField), and the reconstructed neurons were edited in PhotoShop (Adobe Systems, San Jose, CA). The putative synaptic contacts were identified under the light microscope as described previously (Buhl et al., 1994; Tamas et al., 1997; Gupta et al., 2000).

\section{Results \\ Dual modes of inhibition and differential modulatory effects of dopamine}

Nonpyramidal interneurons were identified under infrareddifferential interference contrast videomicroscopy by their typical round or oval soma and bipolar or multipolar dendritic orientations, and further distinguished by their narrow action potentials and fast repolarization (Kawaguchi, 1995; Kawaguchi and Kubota, 1997; Gibson et al., 1999). Thirty-five NP-P pairs were successfully recorded before, during, and after dopamine application. Per the criteria described by Kawaguchi (1995) and Gibson et al. (1999), we classified all of our presynaptic interneurons into FS and non-FS groups (Fig. 1 $A-D$ ). FS cells were easily recognized according to their narrow action potentials, deep and brief afterhyperpolarization, and high firing rates with little or no frequency adaptation (Fig. 1A). Non-FS cells formed a more diverse group, which included RS (Fig. $1 B$ ), LTS, and LS cells (see Materials and Methods). Twenty-one of the 35 presynaptic interneurons $(60 \%)$ examined were of the FS type, whereas the remaining 14 cells were classified as non-FS cells (seven RSs, six LTSs, and one LS).

Application of dopamine over a range of concentrations, either by puff or bath perfusion, revealed remarkably dichotomous effects on the IPSP amplitudes of FS and non-FS NP-P connections (holding potential, $-52.9 \pm 0.06 \mathrm{mV}$; reversal potential, $-70.2 \pm 0.56 \mathrm{mV}$ ) (Figs. 2A,B, 3A,B). Dopamine significantly decreased the IPSP amplitude in each of 17 FS NP-P connections $(94.4 \%)$ and increased it in one such pair $(n=18 ;-38.6 \% \pm$ $5.30 ; p<0.001)$. Because dopamine enhances the excitability of FS interneurons and increases spontaneous GABA release (Zhou and Hablitz, 1999), it is possible that the depressing effects of dopamine on IPSPs in pyramidal neurons could have resulted from stimulation of $\mathrm{GABA}_{\mathrm{B}}$ receptors on interneuronal terminals. To examine this possibility, we iontophoretically applied GABA (10 mM, 50-100 nA, 10-20 msec) on six pyramidal cell somata. Consistent with a previous study (Gonzalez-Islas and Hablitz, 2001), the amplitudes of GABA-induced current ob- 
A
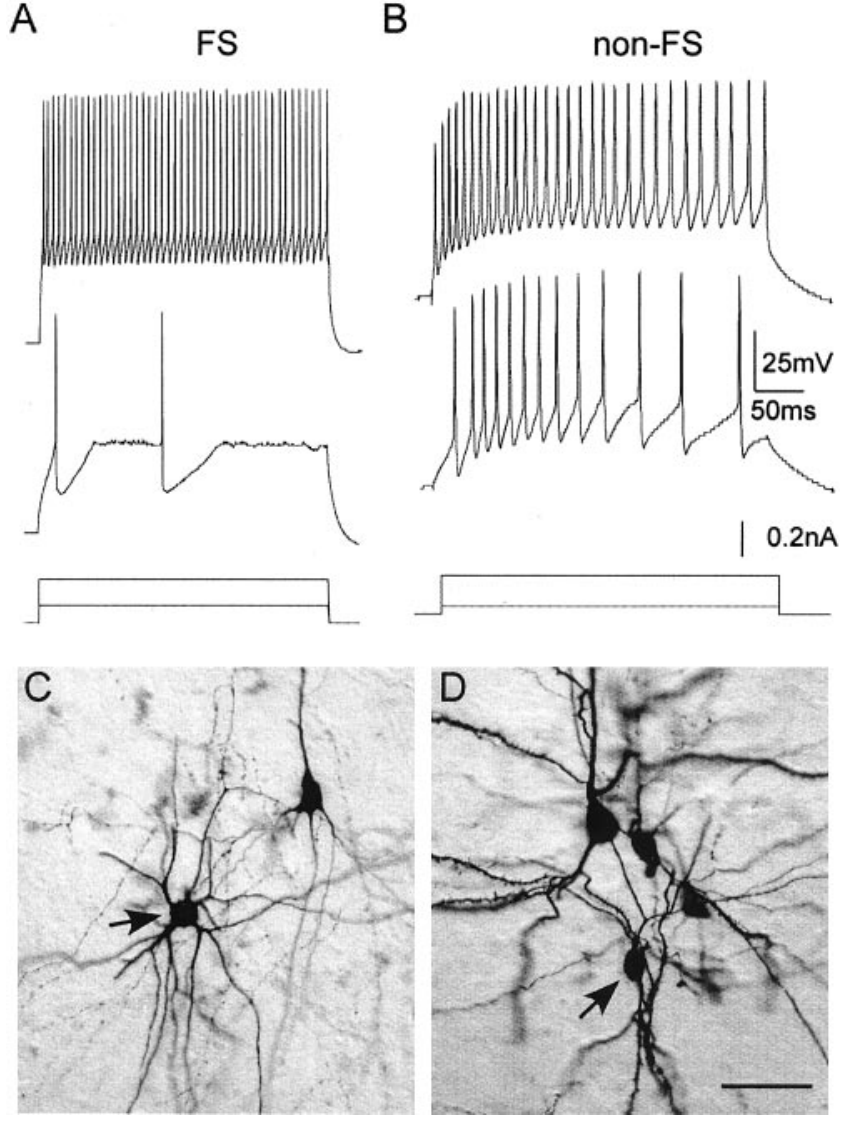

Figure 1. Firing patterns of two types of interneurons. $A, B$, Current-clamp recordings from an FS cell $(A)$ and a non-FS interneuron $(B)$ during injection of depolarizing current pulses of two intensities. Note the short afterhyperpolarization after a spike and the repetitive highfrequency firing without adaptation in the FS cell. In contrast, the regular spiking cell $(B)$ has prominent firing adaptation, a wider action potential, and a lower firing frequency. C, D, Samples of biocytin-labeled interneuron-pyramidal cell pairs. The FS interneuron ( $C$, arrow) has clear multiple dendritic processes with characteristics of basket cells, whereas the non-FS interneuron $(D$, arrow) has vertically oriented dendrites characteristic of a double bouquet cell. Scale bar, $50 \mu \mathrm{m}$.

served in pyramidal cells were unchanged by dopamine (data not shown). This result is compatible with a direct effect of DA on GABA release at presynaptic terminals.

In contrast, IPSP amplitude was increased by dopamine in 11 non-FS NP-P pairs $(91.7 \%)$ while decreasing in one $(n=12$, $44.7 \% \pm 9.55 ; p<0.01)$. The remaining five of the 35 pairs studied exhibited little change $(<10 \% ; p>0.05$; ANOVA) (Fig. $2 C-F$, Table 1 ). In addition, under control conditions, the IPSP amplitudes of FS NP-P pairs were significantly higher than those of the non-FS NP-P pairs $(-0.69 \pm 0.10 \mathrm{vs}-0.30 \pm 0.05 \mathrm{mV}$ with failures; $-0.80 \pm 0.10$ vs $0.36 \pm 0.07$ excluding failures; $p<$ 0.001 ) (Figs. $2 C, D, 3 A$ ). Both depressing and enhancing effects recovered within several minutes after washout (2-3 min for puff application; 5-8 min for bath application (Fig. 2C,D,F). Other contrasts between the two classes of interneurons included differences in firing threshold, resting membrane potential, and most importantly, AHP of action potentials (Table 1).

Previous studies of interneuronal diversity in the neocortex and hippocampus have observed higher IPSP amplitudes, faster rise times, and lower failure rates in somatic targeting interneurons than in the dendrite-targeting interneurons, which are subject to dendritic filtering (Freund and Buzsaki, 1996; Miles et al., 1996; Jiang et al., 2000). Because FS cells are primarily thought to
A

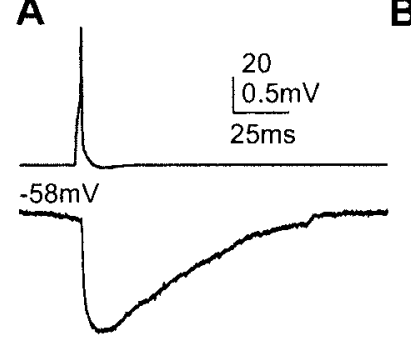

B
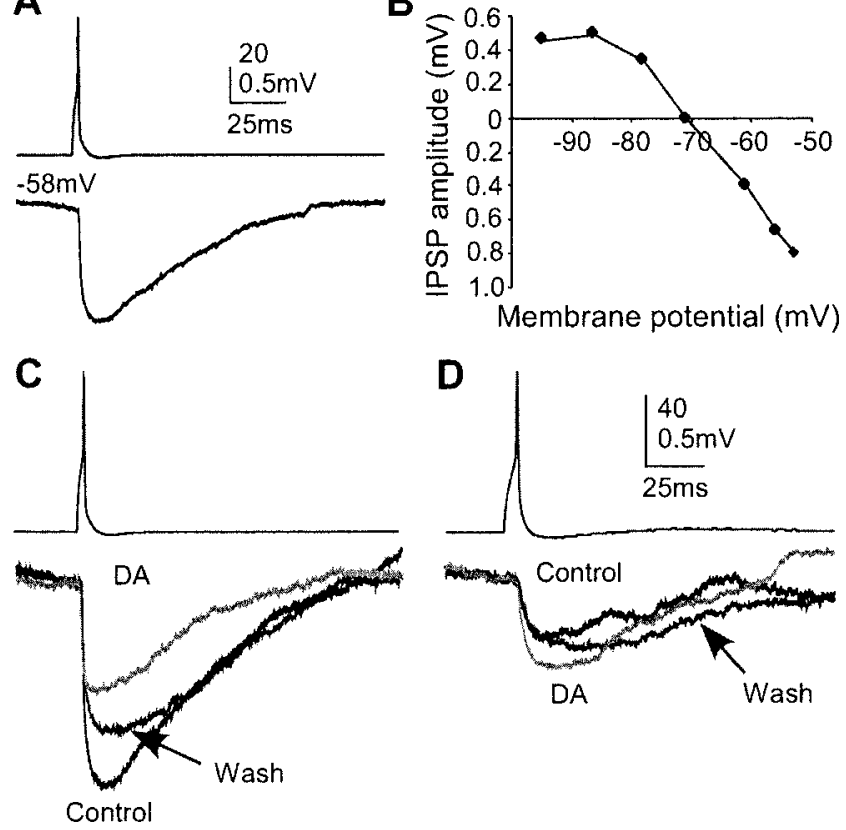

Membrane potential ( $\mathrm{mV})$

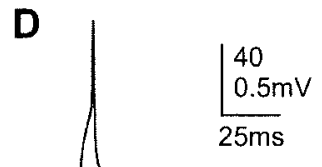

E

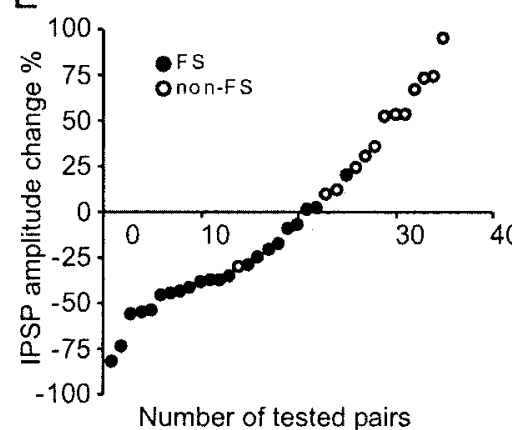

$\mathrm{F}$

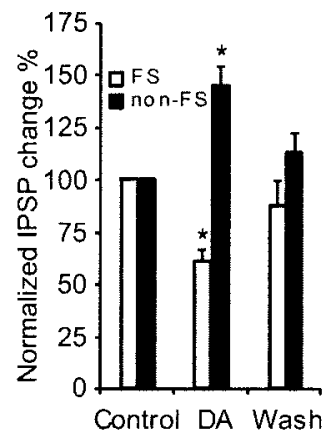

Figure 2. Properties of IPSPs and dopaminergic modulation. A, Action potentials were induced with current injection into the presynaptic interneuron, and the resultant IPSP was recorded in a postsynaptic neuron (average of 20 traces; hold of approximately $-58 \mathrm{mV}$ ). $B$, Graph showing that the reversal potential in this recording condition is approximately $-70 \mathrm{mV}$. $C, D$, Two different classes of dopaminergic modulation: an FS NP-P connection with a large IPSP amplitude and a fast rise time ( () and a non-FS NP-P connection with a smaller IPSP amplitude and slower rise time (D). E, IPSP amplitude change and interneuron classification (FS, filled circle; non-FS, open circle). Most of the FS NP-P pairs exhibit dopamine depression, whereas non-FS NP-P connections show dopamine enhancement. $F$, Summary of dopamine effects in FS and non-FS groups. Dopamine reversibly decreased the IPSP amplitudes of the FS group by $38.6 \%\left(n=18 ;{ }^{*} p<0.001\right)$ but increased IPSP amplitudes in non-FS NP-P pairs by $44.7 \%$ $(n=12 ; p<0.001)$. The two groups were significantly different, but both recovered after a 10 min wash. Note that all averaged IPSPs shown here and in the following figures are without failures.

be somatic targeting or basket-type interneurons and non-FS cells are more likely to be dendritic targeting interneurons (Kawaguchi, 1995; Thomson et al., 1996), we examined rise times and failure rates in the FS NP-P and non-FS NP-P pairs. Particular attention was given to rise time of IPSPs, because this measure is the best biophysical indicator of the mean electronic distance of synaptic inputs and has been shown to correlate with the anatomically determined distance of synaptic contacts (Buhl et al., 1994; Freund and Buzsaki, 1996; Miles et al., 1996; Thomson et al., 1996; Maccaferri et al., 2000; but see Tamas et al., 1997). We found that the FS NP-P connections had significantly shorter rise times (3.44 $\pm 0.54 \mathrm{msec}, 20-80 \%$ amplitude) (see Materials and Methods) compared with the non-FS NP-P group (6.77 \pm 1.11 ; 
A
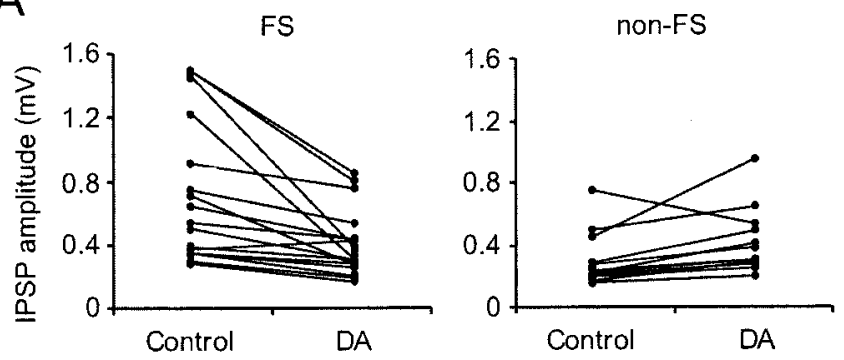

B
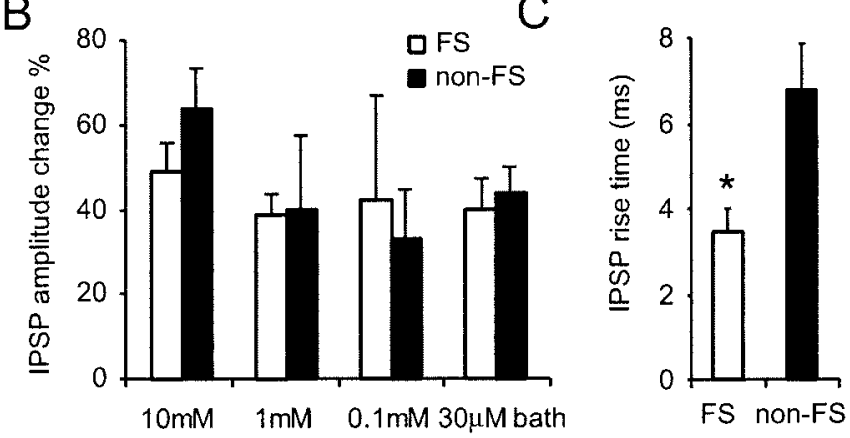

Figure 3. Differential effects of dopamine on FS NP-P and non-FS NP-P connections. $A$, Dopamine effects in FS NP-P $(n=18)$ and non-FS NP-P $(n=12)$ connections indicating that the IPSP amplitudes in the FS group were significantly higher than those in the non- $\mathrm{FS}$ group $(p<0.001) . B$, Similar results were obtained with different dopamine concentrations and application methods in each group. C, The IPSP rise times $(20-80 \%)$ of FS NP-P pairs were significantly shorter than those of non-FS NP-P partners $\left({ }^{*} p<0.01\right)$, indicating a difference of synaptic locations.

$p<0.01$ ) (Figs. $2 C, D, 3 C$, Table 1), suggesting that the two types of inputs arise from synaptic contacts with markedly different soma-dendritic locations on the postsynaptic neuron. Baseline failure rates also differentiated the pairs, being significantly lower in the FS NP-P pairs (average, $11.9 \pm 2.50 \%$ ) than in the non-FS NP-P group (34.6 $\pm 4.37 \%$; $p<0.001$ ) (Fig. $4 A-C$ ) and negatively correlated with IPSP amplitudes $\left(R^{2}=0.448\right)($ Fig. $4 D)$. Moreover, dopamine increased the failure rate threefold in FS NP-P pairs $(p<0.001)$ but produced little or no change in the non-FS NP-P pairs (decrease, 7.1\%; $p=0.2380$ ) (Fig. 4C, Table 1). Finally, failure rate was also negatively correlated with the firing threshold of the interneurons in both circuits $(r=-0.601)$ (data not shown), further implicating subtype specificity in the interneuron and/or connectivity in each of these two groups.

\section{Correlation of dopaminergic modulation with interneuron type and mechanism of action}

The differential effects of dopamine on synaptic failure rate change suggested that dopamine modulation of the two modes of inhibition might act through distinct mechanisms, presynaptic and postsynaptic, respectively (Gao et al., 2001; Gonzalez-Islas and Hablitz, 2001; Seamans et al., 2001). This hypothesis was tested in paired-pulse experiments with $100 \mathrm{msec}$ intervals (10 $\mathrm{Hz}$ ). The majority of FS NP-P pairs (8 of $13 ; 61.5 \%$ ) showed paired-pulse depression (PPD), and only a few (5 of $13 ; 38.5 \%$ ) exhibited paired-pulse facilitation (PPF). Dopamine application significantly increased the paired-pulse ratios in most of these pairs ( 10 of $13 ; p<0.001$ ) (Fig. $5 A, B$, Table 1 ), again supporting a presynaptic mode of action. In contrast, paired-pulse ratios were unchanged in the five non-FS NP-P pairs tested $(n=5 ; p=$ 0.532 ) (Fig. 5B,C, Table 1). Moreover, we computed the CV of
IPSP amplitude in individual recordings for all FS and non-FS NP-P pairs (Kullman, 1994). Although the CV test is also dependent on the signal-to-noise ratio, the advantage of this test is that it is based on detecting a change in a measure reflective of the entire distribution of IPSP amplitudes and is therefore more applicable to small-amplitude IPSPs. As shown in Figure 5, D and $E$, a low CV in FS NP-P connections under control conditions was sharply increased on average by $39.5 \pm 6.81 \%$ with dopamine application $(p<0.001)$, compared with virtually no change $(-8.5 \pm 3.60 \% ; p=0.385)$ in non-FS NP-P pairs. Moreover, the percentage of change in CV was highly correlated with IPSP amplitude change $\left(R^{2}=0.606\right)$ in the FS NP-P pairs but not in the non-FS NP-P connections $\left(R^{2}=0.008\right)$ (Fig. $5 E$ ), further strengthening a differential mechanism of dopamine action on FS and non-FS inhibitory circuitry.

\section{Dopamine receptor involvement in inhibitory modulation}

Dopamine mediates its actions at $\mathrm{D}_{1}$ and $\mathrm{D}_{2}$ receptors. To determine which dopamine receptor was involved in the observed effects, we applied $\mathrm{D}_{1}$ and $\mathrm{D}_{2}$ specific agonists to FS NP-P and non-FS NP-P pairs. As shown in Figure $6, A$ and $B$, the $\mathrm{D}_{1}$ agonist SKF 38393 induced depression of IPSPs to the same degree as dopamine itself in the six FS NP-P pairs tested (decrease 28.6\%; $n=6 ; p<0.05$ ), whereas the $\mathrm{D}_{2}$ agonist quinpirole failed to induce any effect $(n=5 ; p=0.675)$. This finding was confirmed by showing that dopamine failed to induce significant inhibition in FS NP-P pairs in the presence of the $\mathrm{D}_{1}$ antagonist $\mathrm{SCH} 23390$ (decrease, $11.6 \pm 4.78 \% ; n=4 ; p=0.327$ ) (Fig. 6C,D), whereas its action was unaffected by the $\mathrm{D}_{2}$ antagonist raclopride (reduction of $31.7 \pm 6.21 \% ; n=4 ; p<0.05$ ). The effects of these drugs on non-FS NP-P pairs were small and highly variable, and no conclusion could be reached regarding the dopamine receptor(s) involved in their modulation.

Many different receptors have been implicated in the presynaptic modulation of neurotransmitter release in the CNS (for review, see Langer, 1997). Because in our experiments the $D_{1}$ antagonist only partially blocked the depression of FS NP-P transmission caused by dopamine, we tested whether other neurotransmitter receptors might be involved in the modulatory effects observed. The $\mathrm{GABA}_{\mathrm{B}}$ receptor and adrenergic $\alpha-2$ receptors would be highly likely candidates for this effect, because both are known to be located presynaptically (Aoki et al., 1998; Gonchar et al., 2001; Raiteri, 2001). In addition, stimulation of the $\mathrm{GABA}_{\mathrm{B}}$ receptor has been reported to depress IPSPs in the nucleus accumbens (Uchimura and North, 1991; but see Nicola and Malenka, 1997). Therefore, we applied the $\mathrm{GABA}_{\mathrm{B}}$ antagonist CGP55845 at a concentration $(1 \mu \mathrm{M})$ that has been reported previously (Nicola and Malenka, 1997) on three FS NP-P pairs. In none of these pairs was there evidence of an effect on IPSP amplitudes or kinetics, and moreover, bath perfusion of DA (30 $\mu \mathrm{M})$ in the presence of CGP55845 still depressed IPSP amplitudes by $21.5 \pm 7.47 \%(n=3)$ (Fig. $6 E)$. This result is consistent with previous reports by Nicola and Malenka (1997) and Seamans et al. (2001). Both groups also found a lack of effect of $\mathrm{GABA}_{B}$ antagonists on DA actions in the nucleus accumbens and prefrontal cortex, respectively. Noradrenaline and $\alpha$-adrenergic agonists have also been shown to depolarize FS interneurons in the frontal cortex (Kawaguchi and Shindou, 1998). However, we found that the selective $\alpha$-adrenergic antagonists Yohimbine (100 nM) or Prozasin (100 nM) (Marek and Aghajanian, 1999) did not block the depressive action of DA on IPSPs between FS interneurons and pyramidal cells in the four pairs examined. The IPSPs were depressed $24.8 \pm 5.55 \%$ by dopamine in the presence 
Table 1. Properties of IPSPs and presynaptic interneurons in dual recording from PFC inhibitory circuits ${ }^{a}$

\begin{tabular}{|c|c|c|c|c|}
\hline \multirow[b]{2}{*}{ IPSP or interneuron properties } & \multicolumn{2}{|l|}{$\mathrm{FS}(n=18)$} & \multicolumn{2}{|l|}{ Non-FS $(n=12)$} \\
\hline & Control & $\mathrm{DA}$ & Control & DA \\
\hline Amplitude (mV) & $-0.69 \pm 0.10$ & $\begin{aligned}-0.39 & \pm 0.05^{* *} \\
(-38.6 \% & \pm 5.30)\end{aligned}$ & $-0.30 \pm 0.05^{t+t}$ & $\begin{array}{r}-0.42 \pm 0.06^{*} \\
(+44.7 \% \pm 9.55)\end{array}$ \\
\hline $20-80 \%$ rise time (msec) & $3.44 \pm 0.54$ & $3.89 \pm 0.66$ & $6.77 \pm 1.11^{\dagger \dagger}$ & $7.19 \pm 1.84$ \\
\hline Failure rate (\%) & $11.9 \pm 2.50$ & $33.4 \pm 6.05^{* *}$ & $34.6 \pm 4.37^{\dagger \dagger \dagger}$ & $27.5 \pm 3.77$ \\
\hline Normalized paired-pulse ratio (\%) & $\begin{array}{l}100 \\
(n=13)\end{array}$ & $\begin{array}{c}127.7 \pm 17.41^{b * * *} \\
\quad(n=13)\end{array}$ & $\begin{array}{c}100 \\
(n=5)\end{array}$ & $\begin{array}{c}103.6 \pm 5.23 \\
(n=5)\end{array}$ \\
\hline CV & $0.43 \pm 0.04$ & $0.60 \pm 0.04^{* * *}$ & $0.59 \pm 0.04^{\mathrm{ttt}}$ & $0.54 \pm 0.03$ \\
\hline Latency (msec) & $0.86 \pm 0.09$ & $0.93 \pm 0.11$ & $0.76 \pm 0.10$ & $0.82 \pm 0.07$ \\
\hline Decay $(\tau, \mathrm{msec})$ & $65.7 \pm 9.84$ & $57.6 \pm 8.37$ & $53.9 \pm 6.81$ & $55.5 \pm 9.58$ \\
\hline Membrane potential of interneuron (mV) & $-66.4 \pm 1.01$ & $-59.4 \pm 1.48^{* *}$ & $-72.4 \pm 2.37^{\dagger}$ & $-68.4 \pm 2.31^{* *}$ \\
\hline Threshold of interneuron (mV) & $-43.1 \pm 1.21$ & $-44.1 \pm 0.88$ & $-51.6 \pm 1.20^{t+t}$ & $-52.7 \pm 1.76$ \\
\hline Reversal potential (mV) & $-69.3 \pm 0.89$ & & $-71.1 \pm 0.92$ & \\
\hline AHP of interneuron action potential (mV) & $14.0 \pm 0.67$ & & $11.2 \pm 0.66^{\dagger \dagger}$ & \\
\hline Half-width of interneuron action potential (msec) & $0.53 \pm 0.03$ & & $0.64 \pm 0.04^{\dagger}$ & \\
\hline Input resistance of interneuron $(\mathrm{M} \Omega)$ & $72.5 \pm 11.62$ & & $63.8 \pm 15.35$ & \\
\hline Membrane potential of pyramidal neuron (mV) & $61.0 \pm 1.12$ & $60.4 \pm 1.46$ & $59.8 \pm 1.40$ & $58.2 \pm 0.59$ \\
\hline
\end{tabular}

${ }^{a}$ All data are presented as means \pm SE.

${ }^{b}$ Average of both PPD and PPF.

${ }^{*} p<0.05,{ }^{* *} p<0.01,{ }^{* * *} p<0.001$ between control and DA within group.

${ }^{+} p<0.05,{ }^{+t} p<0.01,{ }^{+t+} p<0.001$ between FS NP-P and non-FS NP-P group under control conditions.

of Yohimbine $(n=4)$ (Fig. $6 F)$ and $23.2 \pm 3.42 \%$ in the presence of Prozasin $(n=2)$ (data not shown). These results further support the involvement of $\mathrm{D}_{1}$ presynaptic actions on inhibitory transmission in prefrontal circuitry.

\section{FS and non-FS NP-P pairs correlate with specific morphological characteristics}

The conclusion of physiological analysis was verified by morphological examination of the biocytin-injected interneurons. The processes of eight FS interneurons and six non-FS interneurons were labeled sufficiently well to allow identification of their dendritic and axonal arborizations. Sample biocytin-labeled FS and non-FS NP-P pairs are shown in Figure 7, $A$ and $B$, along with their associated firing pattern and drug effects. The threedimensional reconstruction in Figure $7 A$ reveals that the axon of the FS interneuron formed 14 putative synaptic contacts on the soma and proximal dendrites of its pyramidal cell partner, with an average distance of $83 \mu \mathrm{m}$ from the pyramidal soma. All eight FS interneurons were morphologically identified as basket cells on the basis of their axonal and dendritic arborizations (five large basket cells, one small basket cell, and two nest basket cells). Putative somatic synapses were also found in all of these pairs. The non-FS interneuron shown in Figure $7 B$ exhibited the regular spiking firing pattern. The accompanying reconstruction revealed that it was a bitufted cell with beaded axons that formed 25 putative synaptic contacts primarily on the middle and distal dendrites of the postsynaptic pyramidal neuron with which it interacted. The average distance of these contacts from the soma of this pyramidal cell was $258 \mu \mathrm{m}$, the approximate location of layer $\mathrm{V}$ cells where excitatory thalamic and corticocortical afferents would be expected to be most dense (LeVay and Gilbert, 1976; White, 1989). Six non-FS interneurons that were successfully reconstructed include two bitufted cells, one Martinotti cell, and two double bouquet cells. The one remaining cell from this group was difficult to classify but primarily resembled a nest basket cell as described by Gupta et al. (2000). In contrast to FS interneurons, only one non-FS interneuron axon contacted the soma of a pyramidal cell, and notably in this case, only one putative synapse was observed.

\section{Discussion}

The present study is the first paired recording study of inhibitory transmission in the prefrontal cortex and the first to demonstrate that FS interneurons, which target the perisomatic domain of pyramidal cells, are inhibited by dopamine, whereas non-FS interneurons targeting more distal dendrites are not depressed, but instead show a significant enhancement by this neuromodulator. This difference between FS NP-P and non-FS NP-P pairs was demonstrated electrophysiologically and confirmed by morphological identification of the presynaptic interneuron type. In the process of studying dopamine regulation, we also provide the first direct evidence for perisomatic and peridendritic inhibitory transmission in a cortical area involved in working memory functions, thereby extending the generality of circuit mechanisms initially discovered in other regions of the brain (Freund and Buzsaki, 1996; Somogyi et al., 1998; Maccaferri et al., 2000).

\section{FS and non-FS modes of inhibition in prefrontal cortex}

The classification of interneurons in this study was based on generally accepted criteria for FS interneurons: narrow action potentials, deep afterhyperpolarizations, and high firing frequency with little or no frequency adaptation (Kawaguchi, 1995; Gibson et al., 1999). Although these criteria are similar to those of Kawaguchi (1995) and Gibson et al. (1999) in all critical respects, we, like others, did not observe the slow depolarizing voltage ramp with abrupt onset and offset of action potentials reported by Kawaguchi (1995) and Gibson et al. (1999), indicating that this property may not be an obligatory feature of FS cells (Connors and Gutnick, 1990; Buhl et al., 1996; Thomson et al., 1996). Also, consistent with our previous studies in prefrontal cortex (Krimer and Goldman-Rakic, 2001), the firing frequency of our FS interneurons was considerably lower (100-150 vs $300 \mathrm{~Hz}$ ), and the half-widths of action potentials were wider ( 0.53 vs 0.35 $\mathrm{msec}$ ) than comparable values reported in other species and other cortical areas (Kawaguchi, 1995; Buhl et al., 1996; Gibson et al., 1999). Despite these differences, the validity of our classification is strongly supported by biophysical properties such as rise times. IPSPs evoked from non-FS interneurons had rise times nearly twice as long as those evoked from FS interneurons. Although rise 

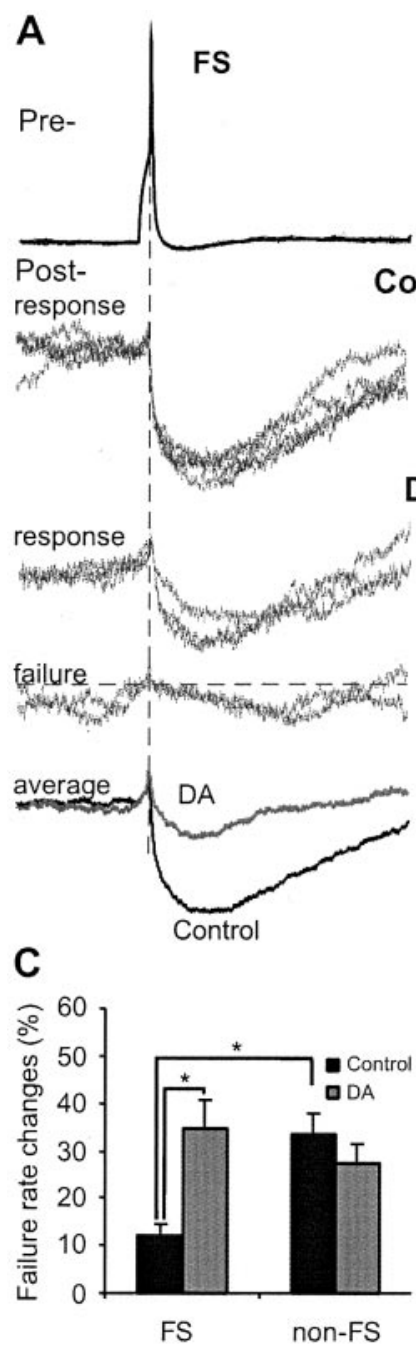

B

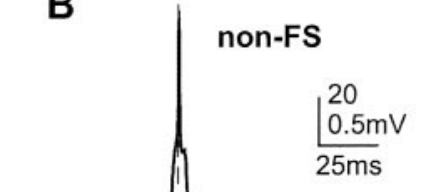

DA
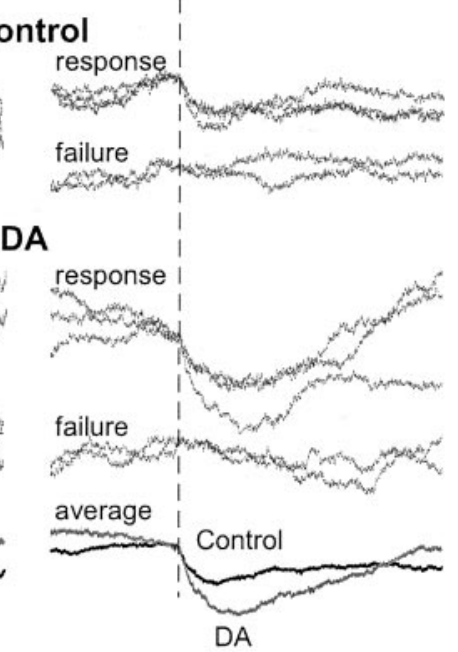

D

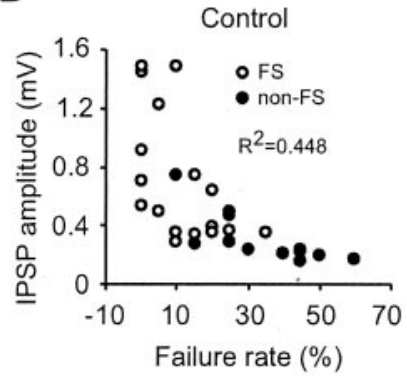

Figure 4. Summary of synaptic failure rates and correlation in two populations of interneurons. $A, B$, Top, Action potentials of five sweeps from a single interneuron of an $F S$ NP-P and a non-FS NP-P pair. Middle, Five representative traces and the average of successful responses from one FS and one non-FS NP-P pair, respectively, showing the synaptic failure before and during dopamine application. Failures were not observed in the FS NP-P pairs during control conditions but did increase under dopamine application. In contrast, in the non-FS NP-P pairs, synaptic failures were observed under control conditions, and their incidence was not affected by DA application. Dashed lines indicate the onset of IPSPs. C, The failure rate was significantly lower in the FS group (11.9 $\pm 2.50 \%)$ than that in the non-FS group $(34.6 \pm 4.37 \% ; p<0.001)$ under control conditions. Moreover, the two groups were differentially modulated by dopamine. The failure rate in the $\mathrm{FS}$ group was significantly increased $21.5 \%$ by dopamine $\left({ }^{*} p<0.01\right)$ compared with little or no change in the non-FS group. D, Failure rates in two groups were negatively correlated with IPSP amplitude $\left(R^{2}=0.448\right)$. This relationship disappeared after application of dopamine (data not shown). Note the electrical coupling between interneuron and pyramidal cell in $A$ and in Figure $6 A$.

time may not be a direct measure of differences in synaptic localization, the large difference in this measure between the two subgroups of inhibitory connections is consistent with a differential localization of their synapses on pyramidal cells, in line with previous structure-function correlations (Buhl et al., 1994; Freund and Buzsaki, 1996; Miles et al., 1996; Thomson et al., 1996; Maccaferri et al., 2000).

Presynaptic versus postsynaptic mechanisms of dopaminergic modulation

Paired recording in this study allowed us to identify the presynaptic member of the recorded pairs and to examine possible dis-
A

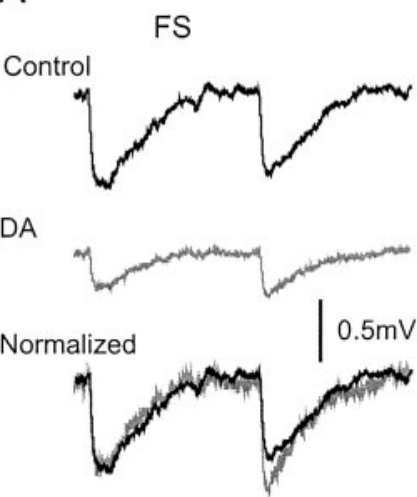

C

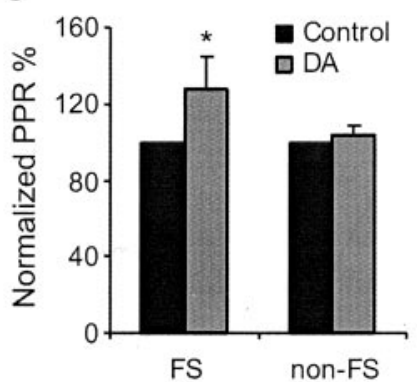

$E$

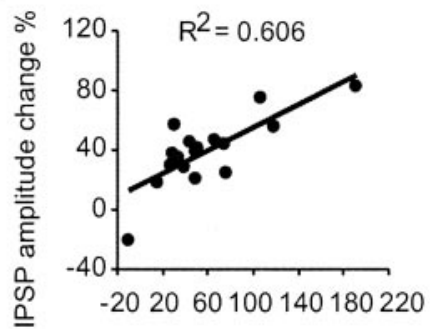

B

D non-FS
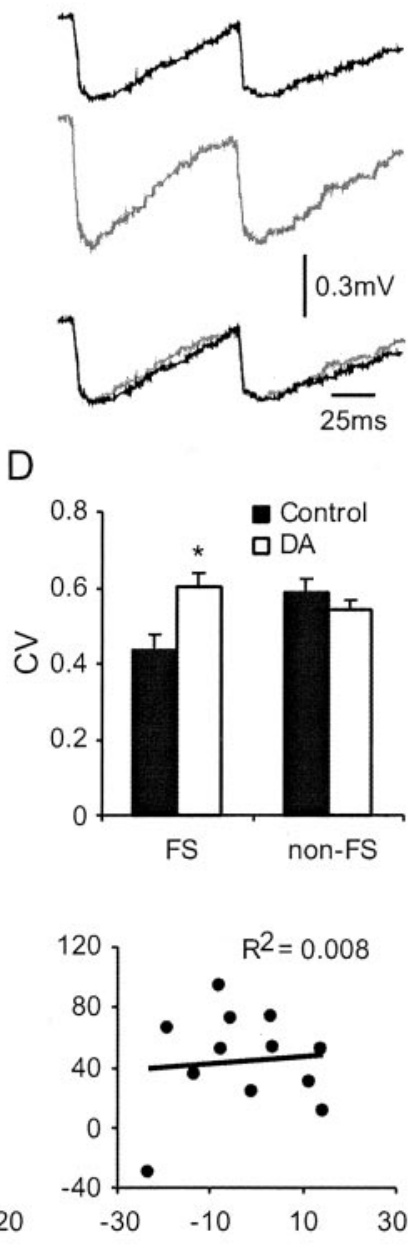

CV change $\%$

Figure 5. Changes in PPR, CV, and correlation between CV and IPSP amplitude change. $A, B$, Paired-pulse ratio change in the FS and non-FS NP-P pairs. A majority of the FS NP-P pairs tested ( $n=13$ ) exhibited paired-pulse depression at $10 \mathrm{~Hz}$. Dopamine increased the ratio of second IPSP to first IPSP as presented in normalized traces in FS NP-P connections but had no clear effect in non-FS NP-P pairs. C, Summary of normalized PPR showing that dopamine significantly increased the ratio by $27.7 \%\left({ }^{*} p<0.001\right)$ in FS NP-P connections; no effect was seen for non-FS NP-P connections. D, Because of the low failure rate and large IPSP amplitude in FS NP-P pairs, the CV of IPSP amplitudes under control conditions was significantly lower than that of non-FS NP-P pairs ( ${ }^{*} p<0.001$ ) and was sharply increased by $39.5 \%$ ( ${ }^{*} p<0.001$ ). In contrast, there was almost no change in the non-FS group. $E$, The percentage of change in the CV in FS NP-P pairs was highly correlated with the percentage of change in IPSP amplitude $\left(R^{2}=0.606\right)$ compared with no significant correlation in non-FS NP-P pairs $\left(R^{2}=0.008\right)$. Note the different scale in the $x$-axis.

tinctions between FS and non-FS inhibitory transmission on their pyramidal cell partners. Indeed, differences between the two types of circuitry were observed not only in IPSP amplitudes and rise times but also in synaptic failure rates, paired-pulse ratios, and CVs. These findings suggested that dopamine-mediated depression of inhibition might involve a presynaptic mechanism, whereas the enhancing effects are possibly postsynaptic. The presynaptic $D_{1}$-mediated depression observed is in agreement with the findings of Gonzalez-Islas and Hablitz (2001), based on extracellular stimulation of prefrontal neurons, but differs from that of Seamans et al. (2001). The discrepancy is undoubtedly attributable to the different conditions in the study by Seamans et 

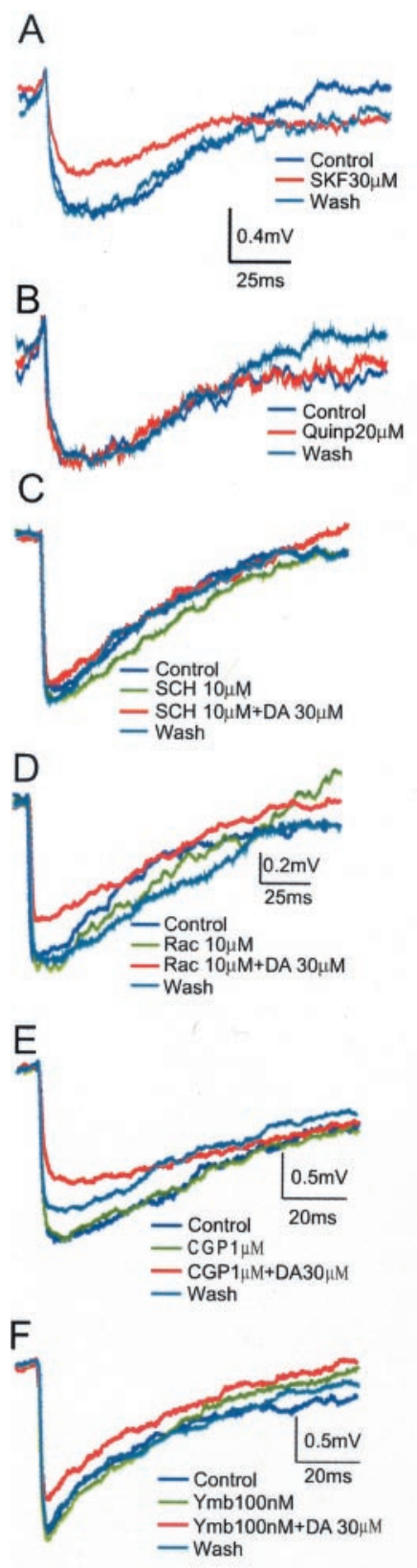

al. (2001), in which the actions of dopamine were not immediate but emerged only several minutes after application and were mediated by $\mathrm{D}_{2}$. A presynaptic mechanism for the inhibitory effect of dopamine on synaptic transmission between FS interneurons and pyramidal cells is also strongly supported by our recent finding that $\mathrm{D}_{1}$ receptors are located on the axon terminals of interneurons (Muly et al., 1998). The lack of effects on the depressive actions of presynaptic autoceptor antagonists of DA ( $\mathrm{GABA}_{\mathrm{B}}$ and $\alpha-2$ adrenergic) also supports $\mathrm{D}_{1}$ presynaptic modulation. Also consistent with our results is a recent study showing that unitary IPSCs between FS interneuron and pyramidal cells in the neocortex are depressed by a kainate receptor agonist (Ali et al., 2001). As in the present study, this effect was also accompanied by an increase in failure rate, $\mathrm{CV}$, and paired-pulse ratio.

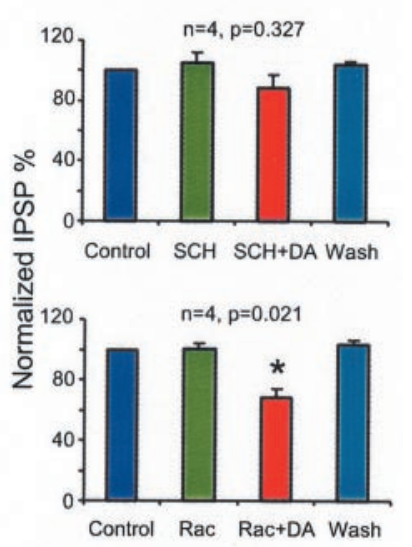

A novel finding in this study is that dopamine enhanced inhibitory transmission in non-FS NP-P connections. This acute effect was observed without indications of altered presynaptic GABA release such as changes in synaptic failure rate, pairedpulse ratio, and CV. Although the mechanism of this effect remains to be determined, we speculate that the enhancing effect of dopamine could occur via a postsynaptic action (e.g., possibly because of a change of the electronic structure of the postsynaptic neuron), a change in the voltage-dependent modulation of local IPSPs, and/or an activation of some receptors such as dopaminergic $\mathrm{D}_{2}$ receptor or $\alpha-1$ adrenoceptor. Dopamine is well known to act on these receptors. It should be noted that non-FS NP-P connections in layer $\mathrm{V}$ are particularly difficult to find $(\sim 1$ in every 50 recordings). Nevertheless, the relative change from control levels in these circuits was substantial $(\sim 45 \%)$ and even larger than the percentage of change observed in FS NP-P connections. Unfortunately, the rarity of these connections curtailed our efforts to characterize the dopamine receptor subtypes involved in this effect. However, the differential effects of dopamine observed do indicate that this neurotransmitter does not simply alter inhibition of pyramidal neurons unidirectionally but selectively modulates GABAergic circuits in the prefrontal cortex. Again, the morphological confirmation that FS interneurons contacted soma and non-FS cells targeted dendrites further supports the circuit dependency of the actions of dopamine in the prefrontal cortex.

\section{Functional implications}

The present findings have implications for understanding the role of dopamine in the working memory functions of the prefrontal cortex because they are expressed in the living animal. The signature functional property of dorsolateral prefrontal neurons recorded in vivo is the capacity for persistent activation in the absence of a preferred stimulus (a property referred to as the memory field of the neuron) (Funahashi et al., 1989). Previous in vivo studies in this laboratory have established that the spatial tuning of these cells requires inhibition of inputs from nonpreferred stimuli (Funahashi et al., 1989; Rao et al., 1999, 2000). The dopamine depression of FS-mediated somatic inhibition could serve to increase the responsivity of a pyramidal neuron to its preferred excitatory input by enhancing the repetitive discharge of sodium-dependent spikes (Freund and Buzsaki, 1996; Yang and Seamans, 1996). Although the mechanism underlying dendritic enhancement of inhibition is less clear, enhancement of inhibition on dendrites could reduce the effectiveness of inputs representing nonpreferred stimuli, a speculation that remains to be examined. Accordingly, dopamine would simultaneously promote the excitation of a pyramidal neuron by its preferred sensory input through depression of somatic inhibition (by FS cells) and at the 


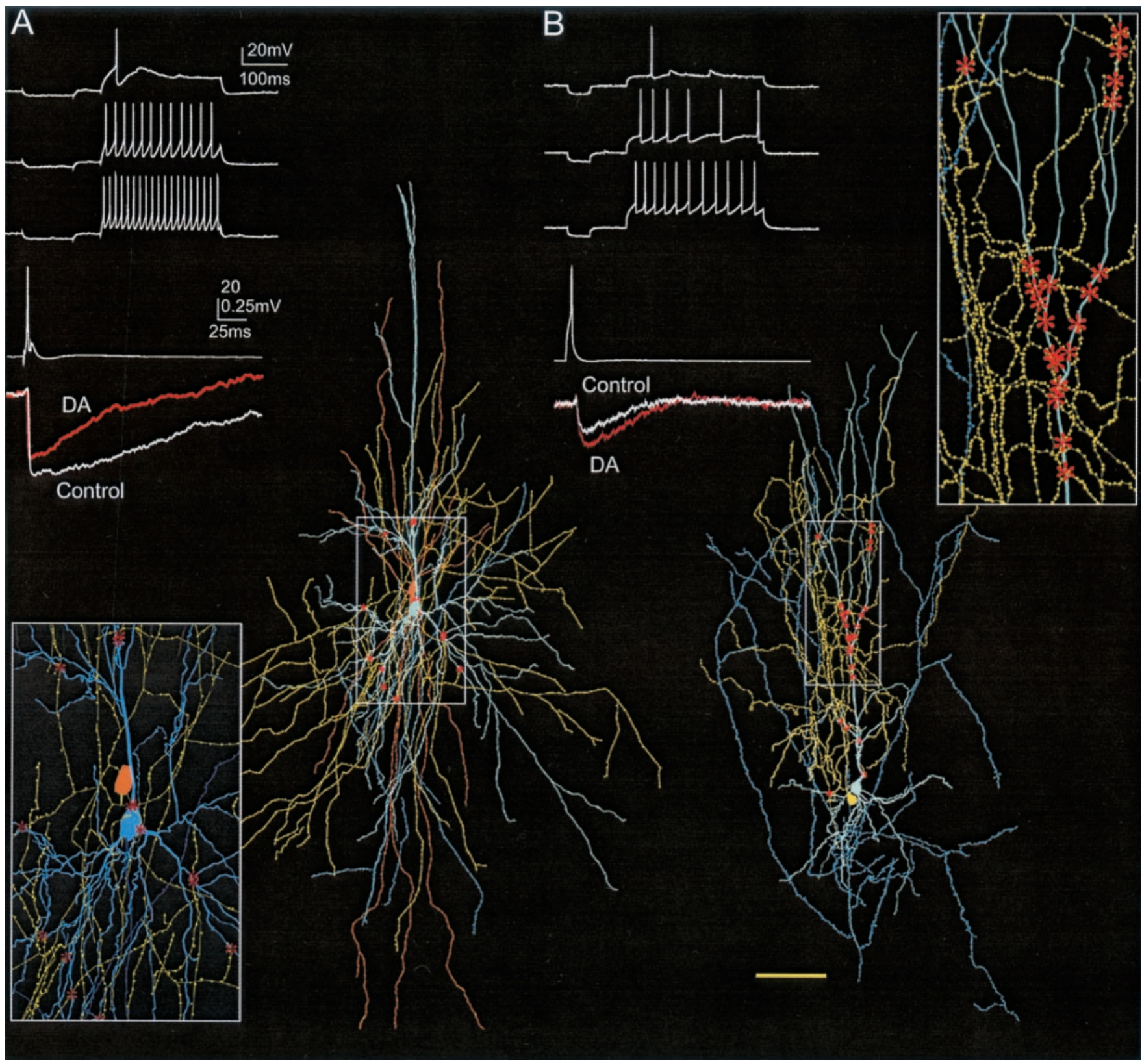

Figure 7. Electrophysiological and morphological characteristics of two neuronal pairs from an FS and non-FS NP-P group, respectively. Three consecutive traces recorded from an FS cell $(A)$ and a non-FS (regular spiking) cell $(B)$ during injection of depolarizing current pulses of three intensities are shown. A single spike induced by narrow current injection in these two presynaptic interneurons elicited correlated IPSPs in the $A$ and $B$ pair, respectively. The two control IPSPs (white line) exhibited distinct properties, as described in Figure 2, whereas DA reduced the IPSP in the FS NP-P pair but enhanced it in the non-FS NP-P pair. Computer reconstructions of these two biocytin-filled interneuron-pyramidal pairs at two magnifications are shown. The interneuron of the FS NP-P pair is a fast-spiking basket cell of the FS type, which forms 14 putative synaptic contacts on the soma and proximal dendrites of the pyramidal neuron (inset, bottom left). The interneuron of the non-FS NP-P pair is an accommodating bitufted cell of the non-FS type with a regular spiking firing pattern. Its beaded axons form 25 putative synaptic contacts primarily on the middle and distal dendrites of the postsynaptic pyramidal neuron with which it interacted (inset, top right). Light blue, Somata and dendrites of the pyramidal neuron; blue, axons of the pyramidal neuron; yellow, axonal arbors of the presynaptic interneuron; orange, dendrites of this interneuron. Scale bar, $100 \mu \mathrm{m} ; A$, inset, $50 \mu \mathrm{m} ; B$, inset, $40 \mu \mathrm{m}$. Asterisks indicate putative synaptic contacts.

same time reduce the effectiveness of nonpreferred stimuli arriving at distal dendrites by shunting inhibition (by non-FS cells) at these sites. Recording studies in behaving monkeys in this laboratory have shown both that FS interneurons themselves are tuned and that some of them have inverted tuning curves as would be suggested by this model (Rao et al., 1999, 2000; Constantinidis et al., 2002). Interneurons have been prominently implicated in disorders such as epilepsy and schizophrenia, and understanding the mechanisms of their modulation may contribute to a better understanding of pathophysiology in these disorders.

\section{References}

Ali AB, Rossier J, Staiger JF, Audinat E (2001) Kainate receptors regulate unitary IPSCs elicited in pyramidal cells by fast-spiking interneurons in the neocortex. J Neurosci 21:2992-2999.

Aoki C, Venhatesan C, Go CG, Forman R, Kurose H (1998) Cellular and subcellular sites of noradrenergic action in the monkey dorsolateral prefrontal cortex as revealed by the immunocytochemical localization of noradrenergic receptors and axons. Cereb Cortex 8:269-277.

Behr J, Gloreli T, Schmitz D, Heinemann U (2000) Dopamine depresses excitatory synaptic transmission onto rat subicular neurons via presynaptic D1-like dopamine receptors. J Neurophysiol 84:112-119. 
Bracci E, Centonze D, Bernardi G, Calabresi P (2001) Dopamine excites fast-spiking interneurons in the striatum. J Neurophysiol 87:2190-2194.

Buhl EH, Halasy K, Somogyi P (1994) Diverse sources of hippocampal unitary inhibitory postsynaptic potentials and the number of synaptic release sites. Nature 368:823-828.

Buhl EH, Szilagyi T, Halasy K, Somogyi P (1996) Physiological properties of anatomically identified basket and bistratified cells in the CA1 area of the rat hippocampus in vitro. Hippocampus 6:294-305.

Connors BW, Gutnick MJ (1990) Intrinsic firing patterns of diverse neocortical neurons. Trends Neurosci 13:99-104.

Constantinidis C, Williams GV, Goldman-Rakic PS (2002) A role for inhibition in shaping the temporal flow of information in prefrontal cortex. Nat Neurosci 5:175-180.

Davis KL, Kahn RS, Ko G, Davidson M (1991) Dopamine in schizophrenia: a review and reconceptualization. Am J Psychiatry 148:1474-1486.

Freund TF, Buzsaki G (1996) Interneurons of the hippocampus. Hippocampus 6:347-470.

Funahashi S, Bruce CJ, Goldman-Rakic PS (1989) Mnemonic coding of visual space in the monkey's dorsolateral prefrontal cortex. J Neurophysiol 61:331-349.

Gao WJ, Krimer LS, Goldman-Rakic PS (2001) Presynaptic regulation of recurrent excitation by D1 receptors in prefrontal circuits. Proc Natl Acad Sci USA 98:295-300.

Gibson JR, Beierlein M, Connors BW (1999) Two networks of electrically coupled inhibitory neurons in neocortex. Nature 402:75-79.

Gonchar Y, Pang L, Malitschek B, Bettler B, Burkhalter A (2001) Subcellular localization of GABA(B) receptor subunits in rat visual cortex. J Comp Neurol 431:182-197.

Gonzalez-Islas C, Hablitz JJ (2001) Dopamine inhibition of evoked IPSCs in rat prefrontal cortex. J Neurophysiol 86:2911-2918.

Gotham AM, Brown RG, Marsden CP (1988) "Frontal" cognitive function in patients with Parkinson's disease "on" and "off" levodopa. Brain 111:299-321.

Gupta A, Wang Y, Markram H (2000) Organizing principles for a diversity of GABAergic interneurons and synapses in the neocortex. Science 287:273-278.

Jiang L, Sun S, Nedergaard M, Kang J (2000) Paired-pulse modulation at individual GABAergic synapses in rat hippocampus. J Physiol (Lond) 523:425-439.

Kawaguchi Y (1995) Physiological subgroups of nonpyramidal cells with specific morphological characteristics in layer II/III of rat frontal cortex. J Neurosci 15:2638-2655.

Kawaguchi Y, Kubota Y (1997) GABAergic cell subtypes and their synaptic connections in rat frontal cortex. Cereb Cortex 7:476-486.

Kawaguchi Y, Shindou T (1998) Noradrenergic excitation and inhibition of GABAergic cell types in rat frontal cortex. J Neurosci 18:6963-6976.

Krimer LS, Goldman-Rakic PS (2001) Prefrontal microcircuits: membrane properties and excitatory input of local, medium, and wide arbor interneurons. J Neurosci 21:3788-3796.

Krimer LS, Jakab RL, Goldman-Rakic PS (1997) Quantitative threedimensional analysis of the catecholaminergic innervation of identified neurons in the macaque prefrontal cortex. J Neurosci 17:7450-7461.

Kullman DM (1994) Amplitude fluctuations of dual-component EPSCs in hippocampal pyramidal cells: implications for long-term potentiations. Neuron 12:1111-1120.

Langer SZ (1997) 25 years since the discovery of presynaptic receptors: present knowledge and future perspectives. Trends Pharmacol Sci 18:95-99.

LeVay S, Gilbert CD (1976) Laminar patterns of geniculocortical projection in the cat. Brain Res 113:1-19.

Maccaferri G, Roberts JDB, Szucs P, Cottingham CA, Somogyi P (2000) Cell surface domain specific postsynaptic currents evoked by identified GABAergic neurons in rat hippocampus in vitro. J Physiol (Lond) 524:91-116.
Marek GJ, Aghajanian GK (1999) 5-HT-2A receptor or alpha-1 adrenoceptor activation induces excitatory postsynaptic currents in layer $\mathrm{V}$ pyramidal cells of the medial prefrontal cortex. Eur J Pharmacol 367:197-206.

Mattay VS, Tessitore A, Callicott JH, Bertolino A, Goldberg TE, Chase TN, Hyde TM, Weinberger DR (2002) Dopaminergic modulation of cortical function in patients with Parkinson's disease. Ann Neurol 51:156-164.

Miles R, Toth K, Gulyas AI, Hajos N, Freund TF (1996) Differences between somatic and dendritic inhibition in the hippocampus. Neuron 16:815-823.

Muly EC, Szigeti K, Goldman-Rakic PS (1998) $\mathrm{D}_{1}$ receptor in interneurons of macaque prefrontal cortex: distribution and subcellular localization. J Neurosci 18:10553-10565.

Nicola SM, Malenka RC (1997) Dopamine depresses excitatory and inhibitory synaptic transmission by distinct mechanisms in the nucleus accumbens. J Neurosci 17:5697-5710.

Nicola SM, Surmeier J, Malenka RC (2000) Dopaminergic modulation of neuronal excitability in the striatum and nucleus accumbens. Annu Rev Neurosci 23:185-215.

Raiteri M (2001) Presynaptic autoreceptors. J Neurochem 78:673-675.

Rao SG, Williams GV, Goldman-Rakic PS (1999) Isodirectional tuning of adjacent interneurons and pyramidal cells during working memory: evidence for microcolumnar organization in PFC. J Neurophysiol 81:1903-1916.

Rao SG, Williams GV, Goldman-Rakic PS (2000) Destruction and creation of spatial tuning by disinhibition: $\mathrm{GABA}(\mathrm{A})$ blockade of prefrontal cortical neurons engaged by working memory. J Neurosci 20:485-494.

Seamans JK, Gorelova N, Durstewitz D, Yang CR (2001) Bidirectional dopamine modulation of GABAergic inhibition in prefrontal cortical pyramidal neurons. J Neurosci 21:3628-3638.

Sesack SR, Snyder CL, Lewis DA (1995) Axon terminals immunolabeled for dopamine or tyrosine hydroxylase synapse on GABA-immunoreactive dendrites in rat and monkey cortex. J Comp Neurol 363:264-280.

Somogyi P, Tamas G, Lujan R, Buhl EH (1998) Salient features of synaptic organization in the cerebral cortex. Brain Res Brain Res Rev 26:113-135.

Stricker C, Field AC, Redman SJ (1996) Statistical analysis of amplitude fluctuations in EPSCs evoked in rat CA1 pyramidal neurons in vitro. J Physiol (Lond) 490:419-441.

Tamas G, Buhl EH, Somogyi P (1997) Fast IPSPs elicited via multiple synaptic sites by different types of GABAergic neurons in the cat visual cortex. J Physiol (Lond) 500:715-738.

Thomson AM, Deuchars J (1997) Synaptic interactions in neocortical local circuits: dual intracellular recordings in vitro. Cereb Cortex 7:510-522.

Thomson AM, West DC, Hahn J, Deuchars J (1996) Single axon IPSPs elicited in pyramidal cells by three classes of interneurons in slices of rat neocortex. J Physiol (Lond) 496:81-102.

Uchimura N, North RA (1991) Baclofen and adenosine inhibit synaptic potentials mediated by gamma-aminobutyric acid and glutamate release in rat nucleus accumbens. J Pharmacol Exp Ther 258:663-668.

Volkow ND, Gur RC, Wang GJ, Fowler JS, Moberg PJ, Ding YS, Hitzemann R, Smith G, Logan J (1998) Association between decline in brain dopamine activity with age and cognitive and motor impairment in healthy individuals. Am J Psychiatry 155:344-349.

White EL (1989) Cortical circuits, Ed 1. Boston: Burkhauser.

Wilson FA, O'Scalaidhe SP, Goldman-Rakic PS (1994) Functional synergism between putative gamma-aminobutyrate-containing neurons and pyramidal neurons in prefrontal cortex. Proc Natl Acad Sci USA 91:4009-4013.

Xiang Z, Huguenard JR, Prince DA (1998) GABA A receptor-mediated currents in interneurons and pyramidal cells of rat visual cortex. Science 281:985-988.

Yang CR, Seamans JK (1996) Dopamine $\mathrm{D}_{1}$ receptor actions in layers V-VI rat prefrontal cortex neurons in vitro: modulation of dendritic-somatic signal integration. J Neurosci 16:1922-1935.

Zhou FM, Hablitz JJ (1999) Dopamine modulation of membrane and synaptic properties of interneurons in rat cerebral cortex. J Neurophysio 81:967-976. 